\title{
CARACTERÍSTICAS POMOLÓGICAS, CAPACIDAD ANTIOXIDANTE Y ÁCIDO ELÁGICO EN FRAMBUESA (Rubus idaeus L.)
}

\author{
POMOLOGICAL CHARACTERISTICS, ANTIOXIDANT CAPACITY AND \\ ELLAGIC ACID IN RASPBERRY (Rubus idaeus L.)
}

\author{
Diana E. Trujano-Fragoso ${ }^{1 *}$, Antonio Trinidad-Santos ${ }^{1}$, Rosa Ma. López-Romero', \\ Ciro Velasco-Cruz' ${ }^{1}$ Alberto E. Becerril-Román ${ }^{1}$ y Consuelo de J. Cortés-Penagos ${ }^{2}$
}

\begin{abstract}
'Postgrado en Edafología, Campus Montecillo, Colegio de Postgraduados. km 36.5 Carretera México-Texcoco. 56230, Montecillo, Texcoco, Estado de México. ${ }^{2}$ Facultad de Químico Farmacobiología, Universidad Michoacana de San Nicolás de Hidalgo. Tzintzunzan 123, Col. Matamoros. 58240, Morelia, Michoacán. México.
\end{abstract}

*Autor para correspondencia (dania.trujano@colpos.mx)

\section{RESUMEN}

El consumo de frutas y vegetales reduce el riesgo de desarrollar enfermedades crónico-degenerativas, por su alto contenido de sustancias bioactivas. Durante 2013-2014 se cosecharon frutos de frambuesa (Rubus idaeus L.) cvs. Adelita, Erika, Lupita y Polka, en Michoacán y Jalisco, México, para determinar color $(L *, a *, b *, C * y h *)$, firmeza, sólidos solubles totales (SST), acidez titulable (AT), pH del jugo, capacidad antioxidante (CA) y concentración de ácido elágico (AE). Como resultado se encontró que todas las variables físicas y químicas, excepto $\mathrm{CA}$, presentaron diferencias significativas para distintas épocas de cosecha. Se realizó un análisis de regresión para evaluar el efecto del promedio mensual de la temperatura $(T)$ y la humedad relativa (HR) de los sitios, y se encontró que existe una relación positiva significativa entre la T promedio y SST, pH, AE y CA, pero negativa y significativa con AT. La HR sólo tuvo efecto negativo sobre las variables firmeza y SST. Se observaron diferencias significativas en la concentración de AE entre cultivares. Las frutas cosechadas en junio y octubre presentaron mayor concentración de AE. La mayor concentración de AE se encontró en Erika (3.69 $\mu \mathrm{g} \mathrm{g}^{-1}$ de peso fresco de la fruta) y la menor concentración en Adelita $\left(2.26 \mu \mathrm{g} \mathrm{g}^{-1}\right)$. La capacidad antioxidante de todos los cultivares fue mayor a $60 \%$, que es aceptable en la neutralización de los radicales libres.

Palabras clave: Cultivares, nutracéuticos, frutillas, polifenoles.

\section{SUMMARY}

Consumption of fruits and vegetables reduces the risk of developing chronic-degenerative diseases, due to their high content of bioactive substances. During 2013-2014 fruits of raspberry (Rubus idaeus L.) cvs. Adelita, Erika, Lupita and Polka, were harvested at Michoacán and Jalisco, Mexico to determine color $(L *, a *, b *, C *$ and $h *)$, firmness, total soluble solids (TSS), titratable acidity (TA), juice $\mathrm{pH}$, antioxidant capacity (AC) and ellagic acid concentration (EA). As a result, it was found that all the physical and chemical variables, except for $\mathrm{AC}$, showed significant differences at different harvesting times. Regression analysis was performed to evaluate the effect of monthly average temperature ( $\mathrm{T})$ and relative humidity $(\mathrm{RH})$ of the sites, and a positive significant relationship was found between average $\mathrm{T}$ and $\mathrm{TSS}, \mathrm{pH}, \mathrm{EA}$, and $\mathrm{AC}$, but negative and significant between T and TA. RH only had negative effect on firmness and TSS. Significant differences were observed in EA concentrations among cultivars. Fruits harvested in June and October presented higher concentrations of EA. The highest EA concentration was found in Erika $\left(3.69 \mu \mathrm{g} \mathrm{g}^{-1}\right.$ fresh fruit weight), while the lowest concentration was found in Adelita $\left(2.26 \mu \mathrm{g} \mathrm{g}^{-1}\right)$. The antioxidant capacity of all cultivars was greater than $60 \%$, which is acceptable for neutralization of free radicals.

Index words: Cultivars, nutraceuticals, berries, polyphenols.

\section{INTRODUCCIÓN}

El consumo de frutas y vegetales reduce el riesgo de desarrollar enfermedades crónico-degenerativas, por su alto contenido de sustancias bioactivas. Las "berries" frescas y procesadas son una excelente fuente de sustancias nutracéuticas con un alto potencial antioxidante (Lee et al., 2012; Šavikin et al., 2009). Diversas investigaciones han reportado las propiedades benéficas de las "berries" sobre la salud humana; por ejemplo, permiten inhibir afectaciones hepáticas (Liu et al., 2010), tienen alta actividad antioxidante, antiinflamatoria, antimicrobiana (Heinonen, 2007), neuroprotectora (Chen et al., 2012), antiangiogénica (Medda et al., 2015) y anticancerígena (Konić-Ristić et al., 2011).

Por la alta actividad biológica que tienen los polifenoles en la salud, es importante determinar su contenido en los frutos (Bowen-Forbes et al., 2010). Un polifenol de gran interés es el ácido elágico, principalmente por su actividad preventiva y supresora de algunos tipos de cáncer. Las "berries" tienen alto contenido de este compuesto (Bobinaité et al., 2012; Li et al., 2009). De ellas, la frambuesa (Rubus idaeus L.) ha sido empleada durante mucho tiempo en el tratamiento no farmacológico de algunas enfermedades crónicas. En ensayos biológicos con ratas Aiyer et al. (2008) reportaron que la frambuesa roja y el ácido elágico, reducen el daño oxidativo endógeno del ADN, lo que ayuda en la prevención de cáncer.

El valor nutracéutico de frambuesa ha motivado un aumento en el consumo y la producción de esta fruta a nivel mundial. Es por ello que en los últimos años México 
ha incrementado la producción de frambuesa tras la demanda de este producto por países como Estados Unidos de América y algunos de Europa. En el periodo de 2000 a 2014 se incrementó en 11 veces el número de hectáreas establecidas con este cultivo. En Jalisco y Michoacán se encuentra el 76 \% de la producción nacional (SIAP, 2016). En dichos estados se emplean diversos cultivares que fueron previamente evaluados, con el propósito de establecer aquellos que mejor se adaptan a las condiciones ambientales con frutos de calidad para la exportación.

Una de las características más importantes para determinar si un cultivar es deseable o no, es su productividad (Milivojević et al., 2011); sin embargo, es también importante considerar los componentes nutracéuticos, calidad física y sensorial, características que los consumidores y los fitomejoradores toman en cuenta. A nivel mundial, ya se considera el contenido de polifenoles en la selección de genotipos debido a los efectos benéficos en la salud humana (Çekiç y Özgen, 2010; Dragišić et al., 2013).

Aunque México es un productor importante y está realizando trabajos de mejoramiento genético en la producción y calidad de la frambuesa, la caracterización de estos cultivares en el contenido e identificación de polifenoles es aún incipiente; sin embargo, se sabe que los factores genéticos y ambientales son importantes por su influencia en estas características (Anttonen y Karjalainen, 2005; Bobinaité et al., 2012).

El objetivo de esta investigación fue estudiar características físicas y químicas, capacidad antioxidante y la concentración del ácido elágico en los frutos de frambuesa de los cultivares Adelita, Erika, Lupita y Polka en Michoacán y Jalisco, México.

\section{MATERIALES Y MÉTODOS}

\section{Caracterización de los sitios}

Los sitios de muestreo corresponden a plantaciones comerciales de frambuesa seleccionadas con base en la información de los cultivares de interés, áreas de cultivo y accesibilidad a los productores (Cuadro 1). La altitud (msnm) y ubicación geográfica se determinaron mediante un GPS eTrex-Garmin ${ }^{\circledR}$, portátil.

\section{Análisis de suelo}

En cada sitio se tomaron sub-muestras de suelo para formar una muestra compuesta. Se reconoció el orden de suelo (Soil Survey Staff, 1999) y se determinaron las siguientes características: densidad aparente, textura, $\mathrm{pH}$, conductividad eléctrica, materia orgánica, nitrógeno total, fósforo extractable, capacidad de intercambio catiónico y cationes intercambiables (SEMARNAT, 2003).

\section{Muestreos y análisis de fruta}

Durante 2013-2014 se cosecharon mensualmente frutos al azar de cada sitio de estudio para formar una muestra compuesta de $400 \mathrm{~g}$ (frutos de calibre medio-grande, 2.5 $\mathrm{g}$ en promedio, en madurez de cosecha). Éstas se transportaron en cajas con hielo y temporalmente se guardaron en un cuarto frío a $4{ }^{\circ} \mathrm{C}$ en el laboratorio de Fruticultura del Colegio de Postgraduados, Campus Montecillo para determinar en fresco, color, firmeza, sólidos solubles totales, $\mathrm{pH}$, acidez titulable y capacidad antioxidante. El resto de la muestra se mantuvo a $-18{ }^{\circ} \mathrm{C}$ hasta la medición de ácido elágico.

\section{Mediciones pomológicas en frambuesa}

El color de la fruta se determinó con un colorímetro HunterLab D25-PC2 (Reston, Virginia, USA) (McGuire, 1992), según las coordenadas $L * a *, b *, C * y h *$. La firmeza se evaluó con un texturómetro (Marca Force Five, Greenwich, Connecticut, USA) por compresión. A cada fruto se le aplicó fuerza hasta causar el cierre del orificio de la fruta que queda cuando éste se desprende del receptáculo, y el resultado se registró en Newtons (N) (Robbins y Moore, 1991). La acidez titulable se realizó de acuerdo con la metodología de Garner et al. (2008a). Los sólidos solubles totales (SST) se determinaron en un refractómetro digital (Atago® PR-32a, Tokio, Japón) (Garner et al., 2008b) y el $\mathrm{pH}$ del jugo se midió con un potenciómetro (Beckman $\Phi$ 40) (Garner et al., 2008a).

\section{Capacidad antioxidante (CA)}

Su determinación se realizó en un espectrofotómetro (HP-8453 UV-visible, Waldbronn, Germany) con arreglo de diodos, a través del radical estable DPPH (2,2-Difenil-1-picril-hidrazil, Sigma-Aldrich) (Brand-Williams et al., 1995). Para ello, los frutos frescos se molieron en un procesador de alimentos(Oster, 2614, México). Se pesaron $5 \mathrm{~g}$ y se agregaron $5 \mathrm{~mL}$ de agua. Se agitó por 15 min y se centrifugó durante 10 min (Gutiérrez et al., 2007). Del sobrenadante se tomó una alícuota de $100 \mu \mathrm{L}$ y se agregaron $3.9 \mathrm{~mL}$ de DPPH $100 \mu \mathrm{M}$, se agitó y se dejó en oscuridad por 30 min (Kuskoski et al., 2005), se midió la absorbancia a 517 $\mathrm{nm}\left(\mathrm{A}_{30}\right)$ y el $\mathrm{A}_{0}$ se determinó con el DPPH (Kim et al., 2002). La capacidad antioxidante se calculó con la siguiente fórmula: $C A=\frac{\left(A_{0}-A_{30}\right)}{A_{0}} \times 100$ (Faller y Fialho, 2010). 


\begin{tabular}{|c|c|c|c|c|c|c|c|c|c|c|c|}
\hline$\frac{\tilde{E}}{\tilde{E}}$ & $\begin{array}{l}0 \\
3 \\
0 \\
0 \\
\mathbb{3}\end{array}$ & $\begin{array}{l}\stackrel{0}{3} \cdot \frac{0}{3} \\
0 \\
\frac{3}{3}\end{array}$ & 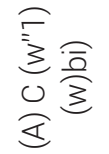 & $\underbrace{\frac{1}{3}}_{0} \frac{\bar{a}}{3}$ & $\begin{array}{l}0 \\
3 \\
0 \\
3\end{array}$ & $\begin{array}{l}\frac{1}{3} \\
0 \\
0 \\
\frac{3}{3}\end{array}$ & $\begin{array}{l}2 \\
3 \\
0 \\
3\end{array}$ & 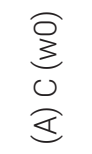 & $\begin{array}{l}0 \\
0 \\
3 \\
0 \\
\mathbb{3}\end{array}$ & $\begin{array}{l}2 \\
3 \\
0 \\
3\end{array}$ & 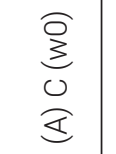 \\
\hline Y & 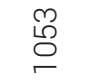 & 옹 & $\underset{\leftarrow}{\mathscr{L}}$ & 。̊ & $\begin{array}{l}\infty \\
\infty \\
\infty \\
10\end{array}$ & 是 & $\underset{\substack{\infty \\
q}}{ }$ & $\stackrel{5}{9}$ & $\stackrel{尺}{尺}$ & $\begin{array}{l}\frac{10}{\sigma} \\
\stackrel{\sigma}{n}\end{array}$ & $\ddot{\mathscr{\theta}}$ \\
\hline 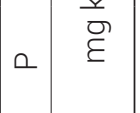 & 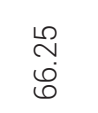 & $\begin{array}{l}\infty \\
\infty \\
0 \\
0\end{array}$ & $\begin{array}{l}\infty \\
\infty \\
\infty \\
\infty\end{array}$ & ம் & $\frac{\kappa}{\sigma o}$ & 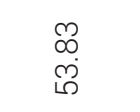 & $\begin{array}{l}\stackrel{\mathscr{P}}{\leftarrow} \\
\leftarrow\end{array}$ & 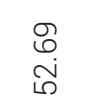 & 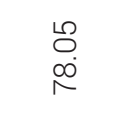 & 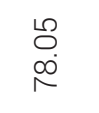 & $\stackrel{\circ}{q}$ \\
\hline$\stackrel{\circ}{\Sigma} \circ^{\circ}$ & $\underset{\sim}{\stackrel{\sim}{+}}$ & $\begin{array}{l}\dot{J} \\
\dot{f}\end{array}$ & $\begin{array}{l}\infty \\
\stackrel{\infty}{+} \\
\dot{\sigma}\end{array}$ & $\begin{array}{l}\dot{J} \\
\dot{+}\end{array}$ & $\check{F}$ & $\stackrel{\circ}{\text { m }}$ & $\stackrel{\sim}{r}$ & $\stackrel{\circ}{r}$ & $\stackrel{\infty}{-}$ & $\stackrel{\sim}{r}$ & $\stackrel{\triangleright}{\sim}$ \\
\hline U & $\stackrel{\sim}{\forall}$ & $\frac{m}{\mathscr{\sigma}}$ & ì. & 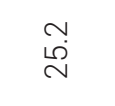 & $\stackrel{+}{\dot{q}}$ & $\begin{array}{l}N \\
\dot{y}\end{array}$ & $\approx$ & $\stackrel{+}{\stackrel{\sim}{\sim}}$ & $\stackrel{丶}{\grave{N}}$ & $\begin{array}{l}m \\
\infty \\
\infty\end{array}$ & $\stackrel{\stackrel{\leftrightarrow}{m}}{\stackrel{m}{m}}$ \\
\hline 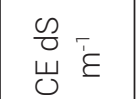 & ले & $\begin{array}{l}\circ \\
\circ\end{array}$ & مُ & $\frac{m}{\circ}$ & $\stackrel{\stackrel{\llcorner}{N}}{\Gamma}$ & ठ̊. & $\begin{array}{l}\stackrel{0}{\circ} \\
\stackrel{0}{0}\end{array}$ & $\begin{array}{l}\circ \\
\circ\end{array}$ & $\stackrel{\infty}{\check{0}}$ & $\stackrel{\widetilde{m}}{0}$ & Nָ \\
\hline 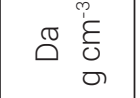 & $\check{r}$ & @̊ & $\stackrel{\circ}{\circ}$ & $\stackrel{\circ}{r}$ & $\check{r}$ & 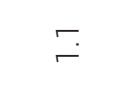 & $\stackrel{\circ}{-}$ & $\stackrel{\circ}{-}$ & $\check{r}$ & $\stackrel{N}{r}$ & $\check{r}$ \\
\hline$\stackrel{I}{\alpha}$ & $\stackrel{N}{N}$ & $\begin{array}{l}\mathscr{\omega} \\
\ddot{0}\end{array}$ & $\underset{0}{\infty}$ & $\stackrel{\infty}{\varphi}$ & $\stackrel{N}{N}$ & $\stackrel{N}{N}$ & @़ & تே & $\stackrel{\bullet}{\sim}$ & $\begin{array}{l}\infty \\
0\end{array}$ & $\hat{\sigma}$ \\
\hline 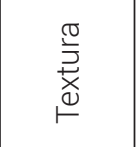 & 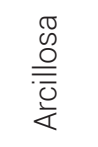 & $\begin{array}{l}\text { D } \\
\text { O } \\
\overline{\bar{O}} \\
\text { 交 }\end{array}$ & 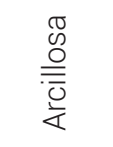 & 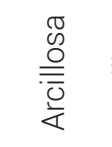 & 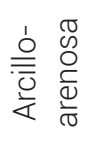 & 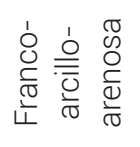 & 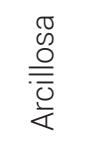 & $\begin{array}{ll}1 & 0 \\
0 & 0 \\
0 & 0 \\
\frac{0}{\pi} & \frac{c}{0} \\
\frac{0}{1} & \frac{d}{\sigma}\end{array}$ & 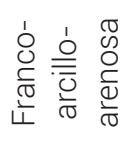 & 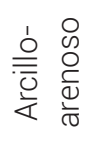 & $\begin{array}{l}\frac{\pi}{\mathscr{D}} \\
\stackrel{0}{0} \\
\overline{\bar{O}} \\
\frac{1}{4}\end{array}$ \\
\hline $\begin{array}{l}\frac{\bar{d}}{0} \\
\frac{0}{0} \\
\frac{\bar{d}}{\frac{0}{D}} \\
\frac{0}{0}\end{array}$ & 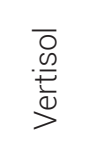 & 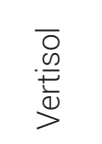 & $\begin{array}{l}\bar{O} \\
\frac{\mathscr{O}}{\bar{O}} \\
\Sigma\end{array}$ & $\begin{array}{l}\bar{D} \\
\frac{.0}{\bar{O}} \\
\sum\end{array}$ & 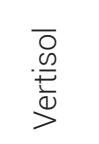 & 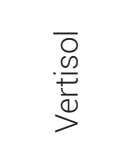 & $\begin{array}{l}\bar{D} \\
\frac{\mathscr{O}}{\bar{O}} \\
\bar{\Sigma}\end{array}$ & 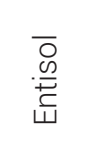 & $\begin{array}{l}\bar{D} \\
\stackrel{.0}{D} \\
\dot{\vec{w}}\end{array}$ & 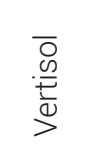 & 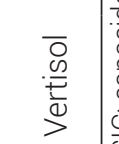 \\
\hline$\stackrel{0}{0}$ & 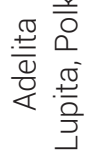 & 胥 & 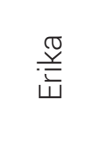 & $\frac{\frac{\pi}{ \pm}}{\frac{\pi}{4}}$ & 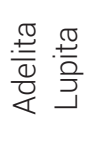 & 蒡 & 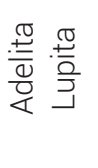 & 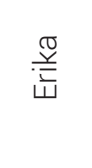 & 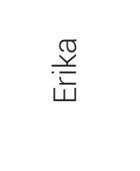 & 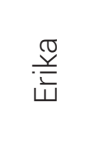 & 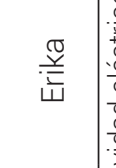 \\
\hline$\pm \frac{\varepsilon}{\Sigma}$ & $\begin{array}{l}\infty \\
\varrho \\
\llcorner \\
\leftarrow\end{array}$ & $\begin{array}{l}\infty \\
\infty \\
\infty \\
\sim\end{array}$ & $\underset{\perp}{\stackrel{\infty}{\perp}}$ & $\underset{\stackrel{O}{\perp}}{\sim}$ & $\begin{array}{l}\infty \\
0 \\
\varnothing\end{array}$ & ָે & $\bar{\varnothing}$ & $\stackrel{N}{N}$ & $\stackrel{m}{\omega}$ & $\begin{array}{l}\hat{0} \\
\stackrel{\circ}{\circ}\end{array}$ & 8 \\
\hline $\begin{array}{l}\frac{.}{0} \\
\frac{0}{0} \\
\frac{0}{0} \\
\frac{0}{D}\end{array}$ & 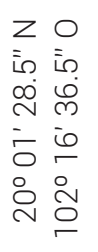 & 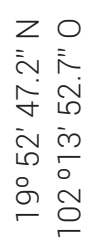 & 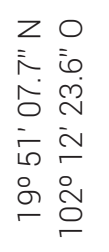 & 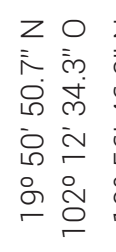 & 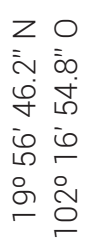 & 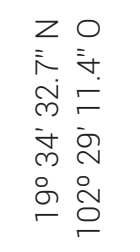 & 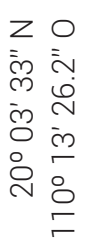 & 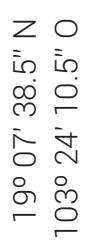 & 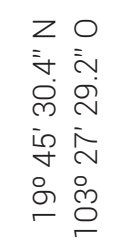 & 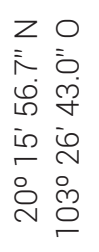 & 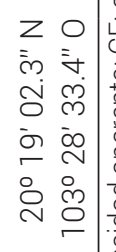 \\
\hline 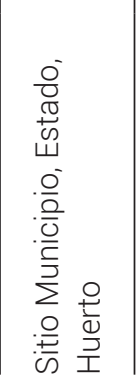 & 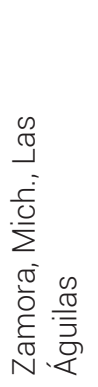 & 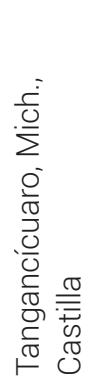 & 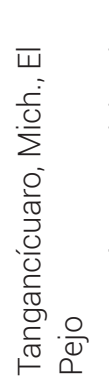 & 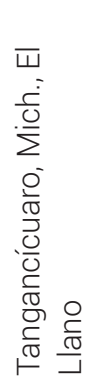 & 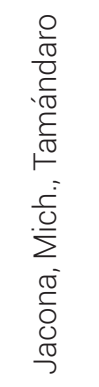 & 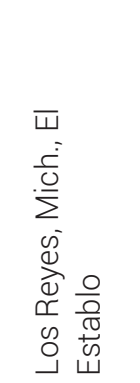 & 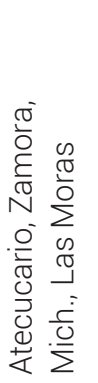 & 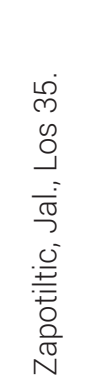 & 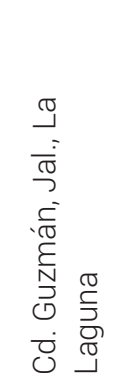 & 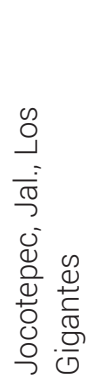 & 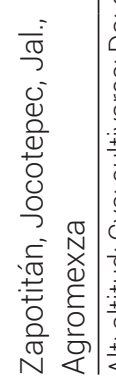 \\
\hline
\end{tabular}




\section{Ácido elágico (AE) \\ Reactivos y materiales}

Se preparó una disolución patrón de $842 \mu \mathrm{g} \mathrm{g}^{-1}$ de AE ( $\geq 95 \%$, Sigma-Aldrich) en dimetil sulfóxido (99.9\% ACS Meyer). La cuantificación del AE se realizó mediante calibración absoluta con estándares diluidos en metanol (MEOH) y preparados diariamente (de 1 a $20 \mu \mathrm{g} \mathrm{g}^{-1}$ ). $\mathrm{MEOH}$ y acetonitrilo (ACN) fueron grado HPLC (Baker) y el $\mathrm{HCl}$ se adquirió con Fermont (34.7\%). Todos los estándares se conservaron a $4^{\circ} \mathrm{C}$. El agua deionizada fue obtenida de un sistema de purificación Simplicity® UV. El $\mathrm{KH}_{2} \mathrm{PO}_{4}$ fue grado analítico (Merck). Para limpiar los extractos se utilizaron cartuchos de extracción en fase sólida Sep-Pak Plus $\mathrm{tC}_{18}$ (900 mg) (Macherey-Nagel). Previa inyección por triplicado $(10 \mu \mathrm{L})$ en el cromatógrafo, las muestras se filtraron en acrodiscos (Titan3, $17 \mathrm{~mm}, 0.2 \mu \mathrm{m}$ ).

\section{Extracción de ácido elágico}

El AE se determinó por el método de Amakura et al. (2000) con algunas modificaciones. En un matraz redondo de $100 \mathrm{~mL}$ se pesaron $10 \mathrm{~g}$ de frutos molidos y homogeneizados (previamente descongelados), se agregaron 30 $\mathrm{mL}$ de $\mathrm{MEOH}$ y se hizo refluir $1 \mathrm{~h}$. Posteriormente la muestra se filtró al vacío (papel filtro Whatman No. 2) y se lavó con $10 \mathrm{~mL}$ de agua. Se realizó una segunda filtración al observarse partículas, el filtrado se concentró a $10 \mathrm{~mL}$ en un rotavapor a $70{ }^{\circ} \mathrm{C}$ (Hahn Shin Scientific Co. Mod HS2001 NS, Gyeonggi, South Korea). Posteriormente se agregaron $100 \mu \mathrm{L}$ de $\mathrm{HCl} 0.1 \mathrm{M}$. Después se limpió la muestra en un cartucho $\mathrm{C}_{18^{\prime}}$, previo acondicionamiento con $10 \mathrm{~mL}$ de $\mathrm{MEOH}$ y $10 \mathrm{~mL}$ de agua. Se cargó toda la muestra, se lavó con $10 \mathrm{~mL}$ de agua y se eluyó con $10 \mathrm{~mL}$ de $\mathrm{MEOH}$, esta fracción se evaporó a sequedad en el rotavapor. Posteriormente se re-disolvió el analito con $\mathrm{MEOH}$, el eluato se recibió en un matraz aforado de $10 \mathrm{~mL}$ y se enrasó, a diferencia de la redisolución con $5 \mathrm{~mL}$ propuesta por Amakura et al. (2000). Los resultados se reportaron en $\mu \mathrm{g} \mathrm{g}^{-1} \mathrm{de}$ ácido elágico (AE) base peso fresco (PF).

\section{Instrumentación}

Un cromatógrafo de líquidos Waters 2695 (Alliance®, USA), acoplado a un detector de absorbancia dual $\lambda$ Waters ${ }^{\circledR} 2487$ (Milford, MA, USA) fue utilizado para el análisis de AE. La separación se realizó en una columna ACE5 $\mathrm{C}_{18}$ de $5 \mu \mathrm{m}(150 \times 4.6 \mathrm{~mm} \mathrm{~d}$. i. $)$ acoplada a una pre-columna de la misma fase estacionaria. El análisis se realizó en modo isocrático, a $360 \mathrm{~nm}$ y a $40 \pm 2{ }^{\circ} \mathrm{C}$. Como fase móvil se usó $\mathrm{ACN}$ y $\mathrm{KH}_{2} \mathrm{PO}_{4} 5 \mathrm{mM}$ (pH 2.5) 82:18 (v/v) a un flujo

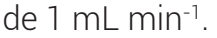

\section{Recuperación}

La recuperación del $\mathrm{AE}$ se evaluó en un cartucho $\mathrm{C}_{18}$. Realizada la extracción a muestras de concentración conocida, se adicionó de 3-6 $\mu \mathrm{g} \mathrm{g}^{-1}$ de AE. El porcentaje de recuperación fue de $89.82 \pm 1.83 \%(n=3)$.

\section{Análisis estadístico}

El análisis estadístico para detectar significancia de las variables dependientes se basó en un modelo de regresión lineal (Weisberg, 2005). Las respuestas de interés se transformaron a logaritmo o raíz cuadrada. Se utilizó un método de selección de variables (stepwise con criterio SBC) para identificar las variables independientes más influyentes (covariables) sobre las variables dependientes, y así lograr modelos simples con una mejor interpretación. Con ellas, se ajustó un modelo para cada variable de interés (Cuadro 2), mediante el procedimiento GLIMMIX del software SAS 9.4 (SAS Institute, 2012), con el propósito de determinar diferencias entre los promedios por variedad y por fecha de muestreo. La separación de medias se hizo mediante la instrucción LS-means de GLIMMIX con las opciones lines y adjust= Tukey, para asignar letras automáticamente y facilitar la comparación de medias, además de controlar el problema de multiplicidad. Las covariables asumieron su promedio aritmético para la separación de medias.

Para determinar el efecto de las variables independientes temperatura $\left(T,{ }^{\circ} \mathrm{C}\right)$ y humedad relativa $(\mathrm{HR}, \%)$ en las características físicas y químicas de la frambuesa, se realizó un análisis de regresión simple mediante el procedimiento REG del software SAS 9.4. La relación entre una variable respuesta de interés y HR o T muestra la pendiente de regresión simple, y su significancia se evaluó mediante las hipótesis de trabajo versus Ho. Si Ho se rechaza a un a $=0.1$, se concluye que la $H R o T$, según sea el caso, tiene un efecto significativo en la variable dependiente.

\section{RESULTADOS Y DISCUSIÓN}

\section{Efecto de época de muestreo}

Se detectaron diferencias estadísticas significativas entre los meses de muestreo. Esto indica que la época de cosecha tiene influencia en las características físicas y químicas de la fruta, incluida la concentración de ácido elágico, con excepción de la capacidad antioxidante de la fruta (Cuadro 3). Anttonen y Karjalainen (2005), Mazur et al. (2014) y Milivojević et al. (2011), señalaron que la luz, temperatura, amplitud del día, precipitación, condiciones del suelo y época de cosecha, pueden provocar diferencias en las características físicas y contenido de ácidos fenólicos, flavonoides y taninos en los frutos. 
Cuadro 2. Variables respuesta en función de las covariables más importantes.

\begin{tabular}{|c|c|c|c|c|c|c|c|c|c|c|c|c|}
\hline \multirow{2}{*}{\multicolumn{2}{|c|}{ Var. Indep }} & \multicolumn{11}{|c|}{ Variables dependientes } \\
\hline & & $L *$ & $a^{*}$ & $b *$ & $C *$ & $h *$ & $F$ & AT & SST & $\mathrm{pH}$ & $\mathrm{CA}$ & $\mathrm{AE}$ \\
\hline \multicolumn{2}{|c|}{ Intercepto } & 3.15 & 4.22 & 3.06 & 3.14 & 2.35 & 1.16 & 1.42 & 2.34 & 1.33 & 4.41 & 2.05 \\
\hline \multicolumn{2}{|c|}{ Temperatura } & - & -0.03 & - & - & 0.03 & - & - & - & - & - & - \\
\hline \multicolumn{2}{|c|}{ Precipitación } & - & -0.00 & - & - & - & - & - & - & - & - & - \\
\hline \multicolumn{2}{|c|}{ Humedad } & - & - & - & - & - & - & - & - & - & - & - \\
\hline \multicolumn{2}{|c|}{ Altitud } & - & - & -0.00 & - & - & - & - & - & - & 0.00 & - \\
\hline \multirow{4}{*}{ CV } & Adelita & - & 0.17 & - & 0.22 & - & - & - & - & - & - & - \\
\hline & Erika & - & 0.23 & - & 0.25 & - & - & - & - & - & - & - \\
\hline & Lupita & - & 0.31 & - & 0.36 & - & - & - & - & - & - & - \\
\hline & Polka & - & 0.00 & - & 0.00 & - & - & - & - & - & - & - \\
\hline \multirow{7}{*}{$S$} & Molisol & - & - & - & - & - & - & - & - & & - & -0.35 \\
\hline & Entisol & - & - & - & - & - & - & - & - & - & - & 0.03 \\
\hline & Vertisol & - & - & - & - & - & - & - & - & - & - & 0.00 \\
\hline & CE & - & - & - & - & - & - & 0.07 & - & - & - & - \\
\hline & $\mathrm{N}$ & - & - & - & - & - & - & - & 0.05 & - & - & - \\
\hline & K & - & - & -0.11 & - & - & - & - & 0.09 & - & - & - \\
\hline & $\mathrm{Na}$ & - & - & - & - & - & - & - & -0.19 & - & - & -0.26 \\
\hline \multirow{8}{*}{ M } & Oct & - & - & - & - & - & - & 0.00 & 0.00 & 0.00 & - & - \\
\hline & Nov & - & - & - & - & - & - & -0.00 & -0.25 & -0.09 & - & - \\
\hline & Dic & - & - & - & - & - & - & 0.06 & -0.23 & -0.09 & - & - \\
\hline & Feb & - & - & - & - & - & - & -0.11 & 0.02 & -0.04 & - & - \\
\hline & Mar & - & - & - & - & - & - & -0.08 & 0.06 & 0.13 & - & - \\
\hline & Abr & - & - & - & - & - & - & -0.22 & 0.06 & -0.01 & - & - \\
\hline & May & - & - & - & - & - & - & 0.06 & 0.05 & -0.04 & - & - \\
\hline & Jun & - & - & - & - & - & - & 0.01 & 0.59 & -0.10 & - & - \\
\hline
\end{tabular}

†a*, [rojo (+)/verde (-)]; coordenada b* [amarillo (+)/azul (-)]; AT: acidez titulable; CA: capacidad antioxidante; AE: concentración de ácido elágico; SST: sólidos solubles totales; Var. Indep.: variables independientes.; CV: cultivar; S: características del suelo del sitio de colecta; N: nitrógeno total; CE: conductividad eléctrica; K: potasio intercambiable; Na: sodio intercambiable; M: mes de muestreo (octubre 2013 - junio 2014 ).

\section{Firmeza (F)}

Se determinó que la T no tuvo efecto en la firmeza del fruto, no así la HR (Cuadro 4). En el mes de octubre la firmeza fue de $1.04 \mathrm{~N}$ y de $1.83 \mathrm{~N}$ en junio (Cuadro 3). Estos valores se relacionaron significativamente con la $\mathrm{HR}$, Io que significa que a mayor HR la F es menor (Cuadro 4). La pérdida de $F$, en la frambuesa, se da por múltiples factores (Kevers et al., 2014; Sexton et al., 1997). La F comienza a disminuir conforme los frutos maduran (Sexton et al., 1997), aunque también la intensidad de la luz, número de horas al día y época productiva de la planta, pueden provocar diferencias en la F.

\section{Sólidos solubles totales (SST)}

La T y la HR influyeron significativamente en el contenido de SST (Cuadro 4). En los meses más fríos y húmedos (noviembre y diciembre), los valores de SST fueron bajos, menores a $8.2^{\circ}$ Brix (Cuadro 3), mientras que entre marzo y junio, periodo cálido y menos húmedo, alcanzaron hasta $10.9^{\circ}$ Brix (Cuadro 3). La diferencia en ${ }^{\circ}$ Brix de SST entre los meses cálidos y fríos indica un mayor contenido de fotosintatos cuando las temperaturas son más elevadas.

\section{Acidez titulable (AT) y pH}

La T fue el factor que más influyó sobre la AT y pH del 
Cuadro 3. Efecto de la época de muestreo en las variables físicas y químicas.

\begin{tabular}{|c|c|c|c|c|c|c|}
\hline $\begin{array}{l}\text { Mes de } \\
\text { cosecha }\end{array}$ & $F(N)^{+}$ & SST $\left({ }^{\circ} B x\right)^{9}$ & AT $(\%)^{\dagger}$ & $\mathrm{pH}^{++}$ & CA (\%) & $A E\left(\mu g g^{-1}\right)^{+}$ \\
\hline Oct & $1.04 \pm 0.06 \quad b$ & $10.72 \pm 0.08 \mathrm{ab}$ & $2.12 \pm 0.06 \mathrm{abc}$ & $3.83 \pm 0.07 a b$ & $65.08 \pm 0.18 a$ & $4.43 \pm 0.14 a b$ \\
\hline Nov & $1.34 \pm 0.08 a b$ & $8.05 \pm 0.03 b$ & $2.06 \pm 0.02 a b c$ & $3.45 \pm 0.00 b$ & $62.67 \pm 0.11 \mathrm{a}$ & $3.10 \pm 0.17 a b$ \\
\hline Dic & $1.55 \pm 0.03 a b$ & $8.25 \pm 0.02 b$ & $2.29 \pm 0.01 \mathrm{a}$ & $3.47 \pm 0.01 b$ & $60.57 \pm 0.04 a$ & $2.63 \pm 0.12 a b$ \\
\hline Feb & $1.11 \pm 0.04 b$ & $10.33 \pm 0.01 \mathrm{ab}$ & $1.80 \pm 0.03 b c$ & $3.66 \pm 0.00 b$ & $59.95 \pm 0.07 a$ & $2.04 \pm 0.10 b$ \\
\hline Mar & $1.20 \pm 0.06 \mathrm{ab}$ & $10.90 \pm 0.02 \mathrm{a}$ & $1.86 \pm 0.03 b c$ & $4.35 \pm 0.00 \mathrm{a}$ & $60.44 \pm 0.11 a$ & $3.07 \pm 0.13 a b$ \\
\hline Abr & $1.45 \pm 0.01 \mathrm{ab}$ & $10.62 \pm 0.02 \mathrm{ab}$ & $1.57 \pm 0.03 c$ & $3.75 \pm 0.00 b$ & $62.90 \pm 0.07 a$ & $3.02 \pm 0.14 a b$ \\
\hline May & $1.27 \pm 0.07 \mathrm{ab}$ & $10.18 \pm 0.05 a b$ & $2.39 \pm 0.02 \mathrm{a}$ & $3.64 \pm 0.01 b$ & $61.62 \pm 0.07 a$ & $2.81 \pm 0.08 \mathrm{ab}$ \\
\hline Jun & $1.83 \pm 0.05 \mathrm{a}$ & $10.96 \pm 0.05 a$ & $2.22 \pm 0.03 a b$ & $3.42 \pm 0.00 b$ & $62.11 \pm 0.11 a$ & $4.70 \pm 0.13 a$ \\
\hline
\end{tabular}

Medias con letras iguales en las columnas no son estadísticamente diferentes (Tukey, 0.05). F: firmeza; SST: sólidos solubles totales; AT: acidez titulable; pH: pH de fruto; CA: capacidad antioxidante; AE: concentración de ácido elágico. La separación de medias fue realizada con los datos en escala logarítmica (`) o raíz cuadrada $\left({ }^{+}\right)$. Los datos se presentan en la escala en que fueron medidos.

Cuadro 4. Efecto de cada aumento unitario de la temperatura (T) y humedad relativa (HR) sobre las variables pomológicas en frambuesa.

\begin{tabular}{|c|c|c|c|c|c|c|}
\hline & $F(N)$ & AT (\%) & SST ( $\left.{ }^{\circ} \mathrm{Bx}\right)$ & $\mathrm{pH}$ del fruto & CA (\%) & $A E \mu g g^{-1} P F$ \\
\hline$T$ & NS & -0.01 & 0.03 & 0.01 & 0.01 & 0.05 \\
\hline$H R$ & -0.0077 & NS & -0.004 & NS & NS & NS \\
\hline
\end{tabular}

F: firmeza; SST: sólidos solubles totales; AT: acidez titulable; pH: pH de fruto; CA: capacidad antioxidante; AE: concentración de ácido elágico; NS: Pendiente no significativa $(a=0.1)$.

fruto (Cuadro 4). Como se observó en el análisis anterior, los factores T y HR influyen en las características físicas y químicas de los frutos en distintos grados; sin embargo, su efecto no provoca diferencias que afecten drásticamente sus características pomológicas (Cuadro 3), lo que indica similitud de los genotipos en estas variables, dentro de los intervalos de T y HR evaluados. Tanto para el consumo en fresco como para la industria, los genotipos que se prefieren son aquellos que producen frutos con características estables de producción y calidad, independientemente de las condiciones climáticas (Mazur et al., 2014) bajo el supuesto de que éstas no varían drásticamente.

\section{Capacidad antioxidante}

La T media de cada uno de los meses influyó positivamente sobre la CA (Cuadro 4), aunque el valor $0.01 \%$ de incremento de CA por cada grado de $T$ es muy pequeño y no se reflejó entre los meses de muestreo (Cuadro 3).

\section{Ácido elágico}

La T es un elemento climático que influyó sobre la concentración de AE (Cuadro 4). Wang y Zheng (2001) reportaron que las altas temperaturas, diurnas y nocturnas, en fresa (Fragaria $\times$ ananassa) provocan incrementos en el contenido de fenoles y antocianinas. En febrero, mes más

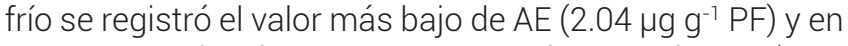
junio, mes más cálido, la concentración fue más alta (4.70 $\mu \mathrm{g} \mathrm{g^{-1 }} \mathrm{PF}$ ) (Cuadro 3). Cabe mencionar que este último valor corresponde a la fruta de la última cosecha (Cuadro 3). Pantelidis et al. (2007) observaron que en las últimas cosechas aumentó significativamente la concentración de fenoles, independientemente de las condiciones climáticas.

Milivojević et al. (2011) señalan que las prácticas agronómicas que se realizan en el cultivo de frambuesa influyen notoriamente en la calidad organoléptica y física de la fruta. Por lo tanto, para una mejor comprensión del efecto de ellas, lo que procedería es un análisis de cada práctica de manejo y otros elementos climáticos sobre la composición química del fruto.

\section{Efecto del cultivar}

\section{Color}

Los valores de luminosidad $(L *)$ estuvieron entre 23.4 y 24.6 y los de hue $(h *)$ de 19.6 a 22.0, sin detectarse diferencias estadísticas significativas entre los cultivares evaluados (Cuadro 5). Estos valores indican que en los cuatro cultivares se observan colores rojos con baja luminosidad, 
los cuales son visualmente más atractivos para los consumidores (Mazur et al. 2014). En el mismo cuadro se muestran los valores de la escala de color CIEL*a*b*, y se observan diferencias estadísticas significativas por cultivar en las coordenadas $a *(21.9-31.4), b *(8.4-11.1)$ y Chroma $(C *)(23.5$ - 33.3), aunque todos los frutos se cosecharon tomando en cuenta el color como referencia para determinar el estado de madurez. Las diferencias en esta variable confirman que el color está determinado genéticamente y es específico para cada cultivar.

En frambuesa, el color se usa para establecer el estado de madurez y frescura; sin embargo, este parámetro está definido por el genotipo y está sujeto a cambios durante el desarrollo y maduración. Un incremento en la intensidad del color significa que hay cambios en el estado de madurez de la fruta. Estos cambios influyen en el número y concentración de componentes fitoquímicos, como las antocianinas (Krüger et al., 2011; Stavang et al., 2015). Después de un periodo de almacenamiento el color cambia significativamente con respecto a los materiales frescos, pero las diferencias en color permanecen entre cultivares (Krüger et al., 2011). Puede observarse que $a *$ y $C$ * de los cultivares Polka y Lupita fueron estadísticamente diferentes (Cuadro 5).

\section{Variables pomológicas}

La CA, F, AT, SST y pH no tuvieron diferencias estadísticas significativas entre los cultivares evaluados (Cuadro 6). El mercado de frutos en fresco exige elevados estándares de calidad (tamaño, color, textura e inocuidad), sobre todo cuando se trata de productos destinados a la exportación, lo cual ha obligado a los productores a establecer cultivares que cumplan con tales requisitos para poder acceder al mercado.

La F puede estar influenciada por otros factores morfológicos como tamaño y número de las drupelas y profundidad de la cavidad del receptáculo; dichas características están determinadas genéticamente (Robbins y Moore 1991). No se observaron diferencias entre cultivares en la F.

Los resultados de AT estuvieron entre 1.94 y $2.07 \%$ y los de SST de 9.34 a $11.03^{\circ} \mathrm{Bx}$ (Cuadro 6). Estas características pueden variar ampliamente entre cultivares. Stavang et al. (2015) señalaron que en el estado correcto para la cosecha del cv. Glen Ample, los frutos cumplen con $9.95 \%$ de sólidos solubles y $2.7 \%$ de AT. Los valores de acidez en el presente trabajo fueron más altos que los reportados por estos autores en esta etapa de madurez de consumo; es decir, la etapa óptima de cosecha; sin embargo, de Ancos et al. (1999) mencionan que frutos con un bajo pH y alta AT están relacionados con mejores características de color.

\section{Capacidad antioxidante}

Una comparación de la CA en diferentes tipos de "berries", menciona que la frambuesa presentó $51.2 \pm 0.3 \%$ y que sólo fue superada por la cereza (Prunus virginiana) que reportó $78.8 \pm 0.5 \%$ (Li et al., 2009). En la variedad Polka se han reportado valores de CA de $42.2 \pm 2.3 \%$ (Bobinaité et al., 2012). En este estudio, los porcentajes de CA estuvieron entre $60.54 \%$ (Lupita) y $65.83 \%$ (Polka). Estos resultados no mostraron diferencias estadísticas entre variedades. Los resultados obtenidos muestran que todos los cultivares tuvieron porcentajes de CA mayores a $60 \%$, por lo que se pueden considerar satisfactorios en la inhibición de radicales libres. La CA puede variar en función del tipo de compuestos fenólicos contenidos en el fruto, la regulación de la síntesis de éstos puede alterar su cantidad y calidad, pues cada tipo de sustancia tiene una CA específica debido a su estructura química única (Rice-Evans et al., 1997). El tipo y cantidad de compuestos presentes en las plantas depende del genotipo y del estado de desarrollo.

\section{Ácido elágico}

La concentración de AE fue la única variable que estuvo influenciada por el cultivar (Cuadro 6). Adelita $\left(2.2 \mu \mathrm{g} \mathrm{g}^{-1}\right.$

Cuadro 5. Efecto del cultivar en el color de los frutos de frambuesa de los estados de Michoacán y Jalisco, México.

\begin{tabular}{|c|c|c|c|c|c|}
\hline \multirow{3}{*}{ Cultivar } & \multicolumn{5}{|c|}{ Color } \\
\hline & $L *$ & $a^{*}$ & $b *$ & \multirow{2}{*}{ Chroma } & \multirow{2}{*}{ Hue } \\
\hline & \multicolumn{3}{|c|}{ Coordenadas } & & \\
\hline Erika & $23.60 \pm 0.03 a$ & $27.59 \pm 0.02 \mathrm{ab}$ & $11.07 \pm 0.04 a b$ & $29.95 \pm 0.02 \mathrm{ab}$ & $22.06 \pm 0.05 a$ \\
\hline Lupita & $23.95 \pm 0.04 a$ & $31.41 \pm 0.02 \mathrm{a}$ & $11.14 \pm 0.03 a$ & $33.37 \pm 0.02 \mathrm{a}$ & $19.62 \pm 0.04 a$ \\
\hline Polka & $24.62 \pm 0.04 a$ & $21.94 \pm 0.08 b$ & $8.48 \pm 0.08 b$ & $23.52 \pm 0.08 b$ & $21.21 \pm 0.02 a$ \\
\hline Adelita & $23.40 \pm 0.02 \mathrm{a}$ & $27.59 \pm 0.04 a b$ & $9.85 \pm 0.03 a b$ & $29.32 \pm 0.04 a b$ & $19.94 \pm 0.03 a$ \\
\hline
\end{tabular}

Medias con letras iguales en las columnas no son estadísticamente diferentes (Tukey, 0.05). La separación de medias se realizó con los datos en escala logarítmica. Los resultados se presentan con los datos sin transformar. 
Cuadro 6. Efecto del cultivar en las variables físicas y químicas de la frambuesa cultivada en los estados de Michoacán y Jalisco, México.

\begin{tabular}{|c|c|c|c|c|c|c|}
\hline Cultivar & $F(N)^{9}$ & AT $(\%)^{9}$ & SST $\left({ }^{\circ} \text { Brix }\right)^{+}$ & pH del fruto" & $\mathrm{CA}(\%)^{+}$ & $A E\left(\mu g g^{-1} P F\right)^{4}$ \\
\hline Erika & $1.41 \pm 0.03 a$ & $1.94 \pm 0.02 \mathrm{a}$ & $10.37 \pm 0.02 \mathrm{a}$ & $3.75 \pm 0.01 \mathrm{a}$ & $61.58 \pm 0.01 a$ & $3.69 \pm 0.07 a$ \\
\hline Lupita & $1.48 \pm 0.04 a$ & $2.07 \pm 0.05 a$ & $9.43 \pm 0.05 a$ & $3.57 \pm 0.01 \mathrm{a}$ & $60.54 \pm 0.01 \mathrm{a}$ & $3.13 \pm 0.14 \mathrm{ab}$ \\
\hline Polka & $1.00 \pm 0.04 a$ & $2.05 \pm 0.03 a$ & $11.03 \pm 0.05 a$ & $3.92 \pm 0.04 a$ & $65.83 \pm 0.03 a$ & $3.02 \pm 0.21 a b$ \\
\hline Adelita & $1.36 \pm 0.03 a$ & $1.98 \pm 0.03 a$ & $9.34 \pm 0.03 a$ & $3.66 \pm 0.01 a$ & $61.31 \pm 0.01 a$ & $2.26 \pm 0.07 b$ \\
\hline
\end{tabular}

Medias con letras iguales en las hileras no son estadísticamente diferentes (Tukey, 0.05). La separación de medias fue realizada con los datos en escala logarítmica $\left({ }^{+}\right)$o raíz cuadrada (`). Los datos se presentan sin transformar. F: firmeza; SST: sólidos solubles totales; AT: acidez titulable; pH: potencial de hidrogeno de fruto; CA: capacidad antioxidante; AE: concentración de ácido elágico.

PF) y Erika $\left(3.6 \mu \mathrm{g} \mathrm{g}^{-1}\right)$ fueron diferentes estadísticamente en esta característica. Estos resultados concuerdan con distintos autores que señalan que el genotipo es un factor determinante en la cantidad de polifenoles en las "berries" (Bobinaité et al., 2012; Çekiç y Özgen, 2010; Mazur et al., 2014; Milivojević et al., 2011). También se observaron variaciones por época de cosecha (Cuadro 3) que afectaron la expresión de los genotipos, en cuanto a la concentración de AE. Existen diferentes situaciones tales como la intensidad de luz, temperatura, deficiencias de agua, daños físicos, entre otros, que pueden estimular el metabolismo secundario y de esta manera provocar una mayor síntesis de compuestos fenólicos, como mecanismo de defensa y protección de las plantas, en todas ellas la enzima fenilalanina amonio liasa (PAL) desempeña un papel importante en la producción de fenoles, puesto que se ha observado mayor síntesis de éstos cuando hay un aumento en su actividad; aun así, su actividad es diferente en modo y proporción, según la especie, debido a las variaciones genéticas (Faller y Fialho, 2010; Reyes et al., 2007).

Aunque existieron diferencias en la concentración de AE entre cultivares, no hubo diferencias estadísticas en capacidad antioxidante, esto indica que AE no es el compuesto que más contribuye a la capacidad antioxidante, dado que las sustancias fenólicas en los diferentes cultivares tienen diversos grados de contribuciones a la actividad antioxidante. Los fenoles pueden presentar distintas actividades biológicas a través de diversos mecanismos moleculares, y no todos ellos están directamente relacionados con la función molecular como antioxidante (Dragišić et al., 2013; Reyes et al., 2007; Zafrilla et al., 2001).

En esta investigación se muestra que la firmeza, SST, AT y pH del fruto varían según la época de cosecha y que también se afecta la concentración de AE. Asimismo, que el cultivar influye en el color de los frutos y la concentración de $A E$, pero no en las otras variables.

\section{CONCLUSIONES}

Todos los cultivares presentaron un porcentaje mayor de $60 \%$ de capacidad antioxidante, lo que ayuda en la neutralización de los radicales libres producto del metabolismo biológico. Los componentes de color $a^{*}$ y $\mathrm{C} *$ fueron diferentes en los cultivares, independientemente que se cosecharon en la misma etapa de madurez comercial. Se encontraron diferencias significativas entre los cultivares en la concentración de ácido elágico, la mayor concentración se observó en el cv. Erika y la menor en Adelita.

\section{AGRADECIMIENTOS}

Este trabajo fue financiado por el Consejo Nacional de Ciencia y Tecnología (CONACYT). El Servicio Meteorológico Nacional (SMN) proporcionó los datos necesarios para calcular los promedios mensuales de temperatura, precipitación y humedad relativa, en los sitios donde se tomaron las muestras. Un agradecimiento especial a los productores de cada huerto de colecta y a Sun-Belle, por las facilidades otorgadas.

\section{BIBLIOGRAFÍA}

Aiyer H. S., M. V. Vadhanam, R. Stoyanova, G. D. Caprio, M. L. Clapper and R. C. Gupta (2008) Dietary berries and ellagic acid prevent oxidative DNA damage and modulate expression of DNA repair genes. International Journal of Molecular Sciences 9:327-341.

Amakura Y., M. Okada, S. Tsuji and Y. Tonogai (2000) High-performance liquid chromatographic determination with photodiode array detection of ellagic acid in fresh and processed fruits. Journal of Chromatography A 896:87-93.

Anttonen M. J. and R. O. Karjalainen (2005) Environmental and genetic variation of phenolic compounds in red raspberry. Journal of Food Composition and Analysis 18:759-769.

de Ancos B., E. Gonzalez and M. P. Cano (1999) Differentiation of raspberry varieties according to anthocyanin composition. Zeitschrift für Lebensmitteluntersuchung und -Forschung A 208:33-38.

Bobinaité R., P. Viškelis and P. R. Venskutonis (2012) Variation of total phenolics, anthocyanins, ellagic acid and radical scavenging capacity in various raspberry (Rubus spp.) cultivars. Food Chemistry 132:1495-1501.

Bowen-Forbes C. S., Y. Zhang and M. G. Nair (2010) Anthocyanin content, antioxidant, anti-inflammatory and anticancer properties of blackberry and raspberry fruits. Journal of Food Composition and Analysis 23:554-560. 
Brand-Williams W., M. E. Cuvelier and C. Berset (1995) Use of a free radical method to evaluate antioxidant activity. LWT Food Science and Technology 28:25-30.

Çekiç C. and M. Özgen (2010) Comparison of antioxidant capacity and phytochemical properties of wild and cultivated red raspberries (Rubus idaeus L.). Journal of Food Composition and Analysis 23:540-544

Chen W., H. Su, Z. Huang, L. Feng and H. Nie (2012) Neuroprotective effect of raspberry extract by inhibiting peroxynitrite-induced DNA damage and hydroxyl radical formation. Food Research International 49:22-26.

Dragišić M. J. J., J. M. Milivojević, M. M. Poledica, M. D. Nikolić and V. M. Maksimović (2013) Profiling antioxidant activity of two primocane fruiting red raspberry cultivars (Autumn bliss and Polka). Journal of Food Composition and Analysis 31:173-179.

Faller A. L. K. and E. Fialho (2010) Polyphenol content and antioxidant capacity in organic and conventional plant foods. Journal of Food Composition and Analysis 23:561-568

Garner D., C. H. Crisosto, P. Wiley and G. M. Crisosto (2008a) Measurement of $\mathrm{pH}$ and titratable acidity. Central Valley Postharvest Newsletter 17:2.

Garner D., C. H. Crisosto, P. Wiley and G. M. Crisosto (2008b) Measurement of soluble solids content. Central Valley Postharvest Newsletter $17 \cdot 4$

Gutiérrez Z. A., L. Ledesma R., I. García G. y 0. Grajales C. (2007) Capacidad antioxidante total en alimentos convencionales y regionales de Chiapas, México. Revista Cubana de Salud Pública 33. http:// www.redalyc.org/articulo.oa?id=21433108 (Marzo 2016).

Heinonen M. (2007) Antioxidant activity and antimicrobial effect of berry phenolics a Finnish perspective. Molecular Nutrition and Food Research 51:684-691.

Kevers C., J. Pincemail, J. O. Defraigne and J. Dommes (2014) Antioxidant capacity of small dark fruits: influence of cultivars and harvest time. Journal of Berry Research 4:97-105.

Kim D. O., K. W. Lee, H. J. Lee and C. Y. Lee (2002) Vitamin C equivalent antioxidant capacity (VCEAC) of phenolic phytochemicals. Journal of Agricultural and Food Chemistry 50:3713-3717.

Konić-Ristić A., K. Šavikin, G. Zdunić, T. Janković, Z. Juranic, N. Menković and I. Stanković (2011) Biological activity and chemical composition of different berry juices. Food Chemistry 125:1412-1417.

Krüger E., H. Dietrich, E. Schöpplein, S. Rasim and P. Kürbel (2011) Cultivar, storage conditions and ripening effects on physical and chemical qualities of red raspberry fruit. Postharvest Biology and Technology 60:31-37.

Kuskoski E. M., A. G. Asuero, A. M. Troncoso, J. Mancini-Filho y R. Fett (2005) Aplicación de diversos métodos químicos para determinar actividad antioxidante en pulpa de frutos. Ciência e Tecnologia de Alimentos 25:726-732

Lee J., M. Dossett and C. E. Finn (2012) Rubus fruit phenolic research: the good, the bad, and the confusing. Food Chemistry 130:785-796.

Li W., A. W. Hydamaka, L. Lowry and T. Beta (2009) Comparison of antioxidant capacity and phenolic compounds of berries, chokecherry and seabuckthorn. Central European Journal of Biology 4:499506

Liu Y., M. Liu, B. Li, J. L. Zhao, C. P. Zhang, L. Q. Lin, H. S. Chen, S. J. Zhang, J. C. Jin, L. Wang, L. J. Li and J. R. Liu (2010) Fresh raspberry phytochemical extract inhibits hepatic lesion in Wistar rat model. Nutrition \& Metabolism 7:84. doi:10.1186/1743-7075-7-84

Mazur S. P., A. Nes, A. B. Wold, S. F. Remberg and K. Aaby (2014) Quality and chemical composition of ten red raspberry (Rubus ideaus
L.) genotypes during three harvest seasons. Food Chemistry 160:233-240

McGuire R. G. (1992) Reporting of objective color measurements HortScience 27:1254-1255

Medda R., O. Lyros, J. L. Schmidt, N. Jovanovic, L. Nie, B. J. Link, M. F. Otterson, G. D. Stoner, R. Shaker and P. Rafiee (2015) Anti inflammatory and anti angiogenic effect of black raspberry extract on human esophageal and intestinal microvascular endothelial cells. Microvascular Research 97:167-180

Milivojević J. M., M. D. Nikolić, J. J. Dragišić M. and D. D. Radivojević (2011) Generative and fruit quality characteristics of primocane fruiting red raspberry cultivars. Turkish Journal of Agriculture and Forestry 35:289-296.

Pantelidis G. E., M. Vasilakakis, G. A. Manganaris and G. Diamantidis (2007) Antioxidant capacity, phenol, anthocyanin and ascorbic acid contents in raspberries, blackberries, red currants, gooseberries and Cornelian cherries. Food Chemistry 102:777-783.

Reyes L. F., J. E. Villareal and L. Cisneros-Zevallos (2007) The increase in antioxidant capacity after wounding depends on the type of fruit or vegetable tissues. Food Chemistry 101:1254-1262.

Rice-Evans C. A., N. J. Miller and G. Paganga (1997) Antioxidant properties of phenolic compounds. Trends in Plant Science 2:152-159.

Robbins J. and P. P. Moore (1991) Fruit morphology and fruit strength in a seedling population of red raspberry. HortScience 26:294-295.

SAS Institute (2012) Statistical Analysis System 9.4. SAS Institute Inc. Cary, NC, USA

Šavikin K., G. Zdunić, T. Janković, S. Tasić, N. Menković, T. Stević and B. Đordević (2009) Phenolic content and radical scavenging capacity of berries and related jams from certificated area in Serbia. Plant Foods for Human Nutrition 64:212-217.

SEMARNAT, Secretaría de Medio Ambiente y Recursos Naturales (2003) Norma Oficial Mexicana (NOM-021-RECNAT-2000), que establece especificaciones de fertilidad, salinidad y clasificación de suelos. Estudios, muestreo y análisis. Diario Oficial de la Federación (Segunda Sección), 23 de abril de 2003. México, D. F. 75 p.

Sexton R., J. M. Palmer, N. A. Whyte and S. Littlejohns (1997) Cellulase, fruit softening and abscission in red raspberry Rubus idaeus L. CV Glen Clova. Annals of Botany 80:371-376.

SIAP, Servicio de Información Agroalimentaria y Pesquera (2016) Cierre de la producción agrícola por año, estado y por cultivo. SAGARPA, México. http://infosiap.siap.gob.mx:8080/agricola_siap_gobmx/AvanceNacionalCultivo.do (Febrero 2016).

Soil Survey Staff (1999) Soil Taxonomy. A Basic System of Soil Classification for Making and Interpreting Soil Surveys. $2^{\text {nd }}$ edition. United States Department of Agriculture and Natural Resources Conservation Service. Washington D. C., USA. 871 p.

Stavang J. A., S. Freitag, A. Foito, S. Verrall, O. M. Heide, D. Stewart and A. Sønsteby (2015) Raspberry fruit quality changes during ripening and storage as assessed by colour, sensory evaluation and chemical analyses. Scientia Horticulturae 195:216-225

Wang S. Y. and W. Zheng (2001) Effect of plant growth temperature on antioxidant capacity in strawberry. Journal of Agricultural and Food Chemistry 49:4977-4982

Weisberg S. (2005) Applied Linear Regression. $3^{\text {rd }}$ edition. John Wiley \& Sons, Inc. Hoboquen, New Jersey, USA. 336 p.

Zafrilla P., F. Ferreres and F. A. Tomás-Barberán (2001) Effect of processing and storage on the antioxidant ellagic acid derivatives and flavonoids of red raspberry (Rubus ideaus) jams. Journal of Agricultural and Food Chemistry 49:3651-3655. 
